\title{
Near-infrared spectroscopy for objectifying cerebral effects of needle and laserneedle acupuncture
}

\author{
Gerhard Litscher ${ }^{\mathrm{a}, *}$ and Detlef Schikora ${ }^{\mathrm{b}}$ \\ ${ }^{a}$ Department of Biomedical Engineering and Research in Anesthesia and Critical Care, University of \\ Graz, Auenbruggerplatz 29, A-8036 Graz, Austria \\ ${ }^{\mathrm{b}}$ Department of Physics and Optoelectronics, University of Paderborn, D-33095 Paderborn, Germany
}

\begin{abstract}
Near infrared spectroscopy (NIRS) has been successfully used in this study to objectify cerebral alterations in oxyhemoglobin and desoxyhemoglobin, due to manual needle acupuncture and laserneedle acupuncture, in 88 healthy volunteers mean age $25.7 \pm 4.0(\bar{x} \pm \mathrm{SD})$ years (19-38 years). Results from Traditional Chinese Acupuncture, Korean and Chinese hand acupuncture, ear acupuncture, combinations of the different acupuncture methods and placebo needling are presented. NIRS seems to be able to shed some light upon the functioning of the different acupuncture methods.
\end{abstract}

Keywords: Near-infrared spectroscopy, needle acupuncture, laserneedle ${ }^{\circledR}$ acupuncture, High-Tech Acupuncture ${ }^{\circledR}$, brain function, Computer-Controlled Acupuncture ${ }^{\circledR}\left(\mathrm{CCA}^{\circledR}\right)$, Computer-Controlled Laserpuncture (CCL)

\section{Introduction}

The connection between puncturing the body with a needle and the reaction at another area of the body is still unclear. However, it has been proven that when particular acupuncture points are stimulated with needles or laser light, specific effects in the brain can be objectivized and quantified with modern cerebral monitoring methods [1-3].

In this present study, we objectivized the systematic changes of oxygenation in the brain [4] using cerebral near-infrared spectroscopy (NIRS), after stimulating acupuncture points according to Traditional Chinese Medicine (TCM), Korean and Chinese hand acupuncture, ear acupuncture and combinations of these different methods.

We analyzed a total of 328 recordings after manual needle and laser needle stimulation from 88 healthy volunteers.

\section{Methods}

\subsection{Near-infrared spectroscopy}

The NIRS method allows the evaluation of changes in cerebral oxygenation through the intact skull and is also gaining importance in acupuncture research because of its non-invasive approach [4-8].

\footnotetext{
*Corresponding author. Tel.: +43 316385 3907, -83907; Fax: +43 316385 3908; E-mail: gerhard.litscher@uni-graz.at; URLs: www.litscher.info, www.litscher.at, www.neuromonitoring.org.
} 


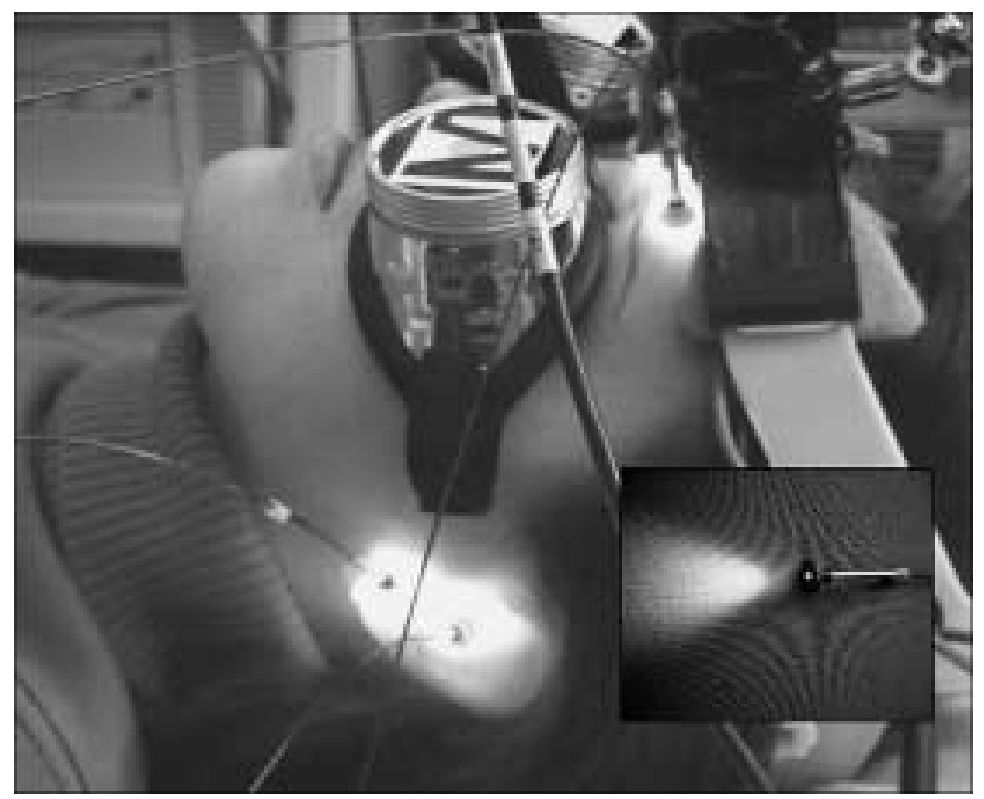

Fig. 1. Test person during laserneedle stimulation and simultaneous registration of NIRS parameters. Right bottom: single active laserneedle and application device.

The NIRO 300 Monitor (Hamamatsu Photonics, Japan) is a new instrument in this field of research. Parameters such as changes in oxyhemoglobin $\left(\Delta \mathrm{O}_{2} \mathrm{Hb}\right)$ and desoxyhemoglobin $(\Delta \mathrm{HHb})$ are determined by Lambert-Beer's principle [4]. The system can measure the absolute value ( $\mu \mathrm{mol})$ of changes in parameters, but not the level (absolute concentration) at which these changes occur (in positive or negative direction). The measurement value is zero, as long as no change in concentration occurs. Placement of the sensor (emitter and near-infrared detectors) on the head with a silicone holder is easy and reproducible. Data output of $\Delta \mathrm{O}_{2} \mathrm{Hb}$ and $\Delta \mathrm{HHb}$ were presented on a color LCD-display and color printer.

In addition to the spectroscopic method, non-invasive, standard monitoring parameters such as blood pressure (Cardiocap ${ }^{\circledR}$ CC-104, Datex Medical Electronics, Hoevelaken, The Netherlands) were determined before, during and after different manners of stimulation.

\subsection{Laserneedle ${ }^{\circledR}$ stimulation}

The Laserneedle ${ }^{\circledR}$-technique represents a new, non-invasive method for optic stimulation of acupuncture points and was first described in literature in 2002 [9,10]. Laserneedle ${ }^{\circledR}$ (Schikora D.: European Patent Nr. PCT/EP 01/08504) acupuncture allows the simultaneous stimulation of individual acupuncture point combinations [9,10]. Variations and combinations of acupuncture at different areas of the body, ear or hand, as performed particularly in this study are possible (compare Fig. 1). Details regarding this method can be found in preceding studies $[9,10]$.

Changes in near-infrared spectroscopic parameters in the frontal region of the brain were continuously registered and analyzed.

\subsection{Healthy volunteers, acupuncture, measurement procedure}

In this study, a total of 328 measurements on 88 healthy volunteers (50 female, 38 male) mean age $25.7 \pm 4.0(\bar{x} \pm \mathrm{SD})$ years (19-38 years) were performed. The study protocol was approved by the ethics 


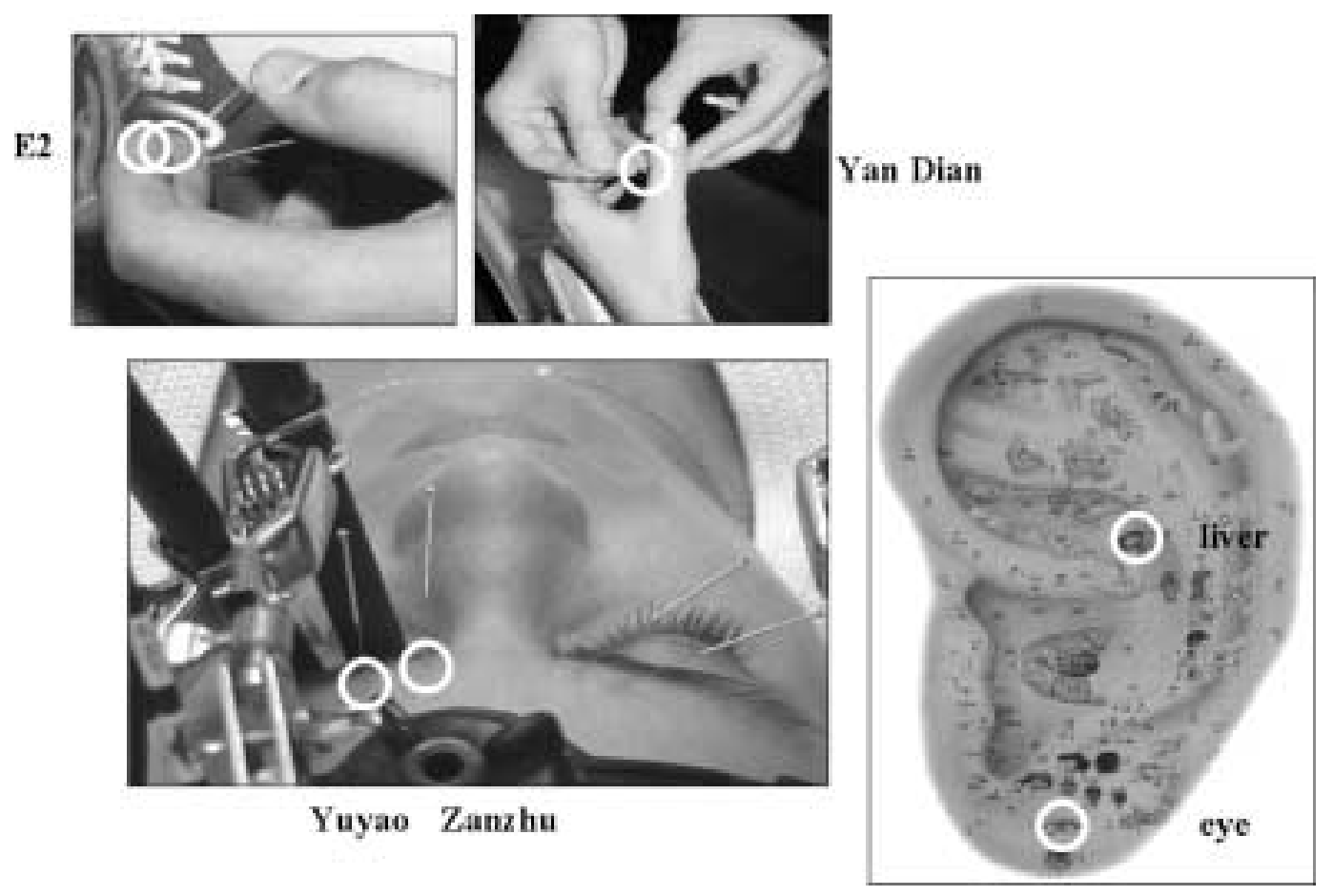

Fig. 2. Acupuncture schemes used in this study.

committee of the University of Graz (11-017) and all test persons gave their written consent. None of the volunteers had visual, neurological or psychological deficits or were under the influence of central nervous system effective drugs.

A maximum of 7 acupuncture points in different measurement series (needle acupuncture and Laserneedle ${ }^{\circledR}$ acupuncture) were investigated. The acupuncture scheme included two acupuncture points from TCM: Zanzhu (localization: at the medial end of the eyebrow, perpendicular to and above the inner corner of the eye, at the foramina of the supraorbital nerve; needling: perpendicular $0.5-0.8$ cun) and Yuyao (localization: at the middle of the eyebrow, perpendicular and above the pupil; needling: inclined 0.3-0.5 cun). In addition, 2 ear acupuncture points (eye and liver: localization see Fig. 2; needling: inclined 0.3 cun) and 2 eye acupuncture points from Korean hand acupuncture (E2: localization see Fig. 2; needling: perpendicular $0.1-0.2$ cun) and one acupuncture point from Chinese hand acupuncture (Yan Dian: localization: on the ulnar side of the middle phalanx of the thumb; needling: inclined 0.2 cun) were included in the study [9-11].

In addition, possible responses in NIRS parameters after needling and stimulating of a placebo point (localization: lateral from the radius 6 cun above the horizontal fold of the wrist exactly on the radial ledge, lateral from the pulmonary meridian) were tested.

The different acupuncture schemes were applied alone and in combination, since preliminary studies indicated that the selection of different combinations also result in different effects in the cerebral parameters to be measured (e.g., bloodflow velocity in the ophthalmic artery) [9-11] (Fig. 2).

Acupuncture points were needled with single-use needles after local desinfection of the skin. We used three different types of needles (body: $0.25 \times 25 \mathrm{~mm}$, Huan Qiu, Suzhou, China; ear: $0.2 \times 13 \mathrm{~mm}$, 

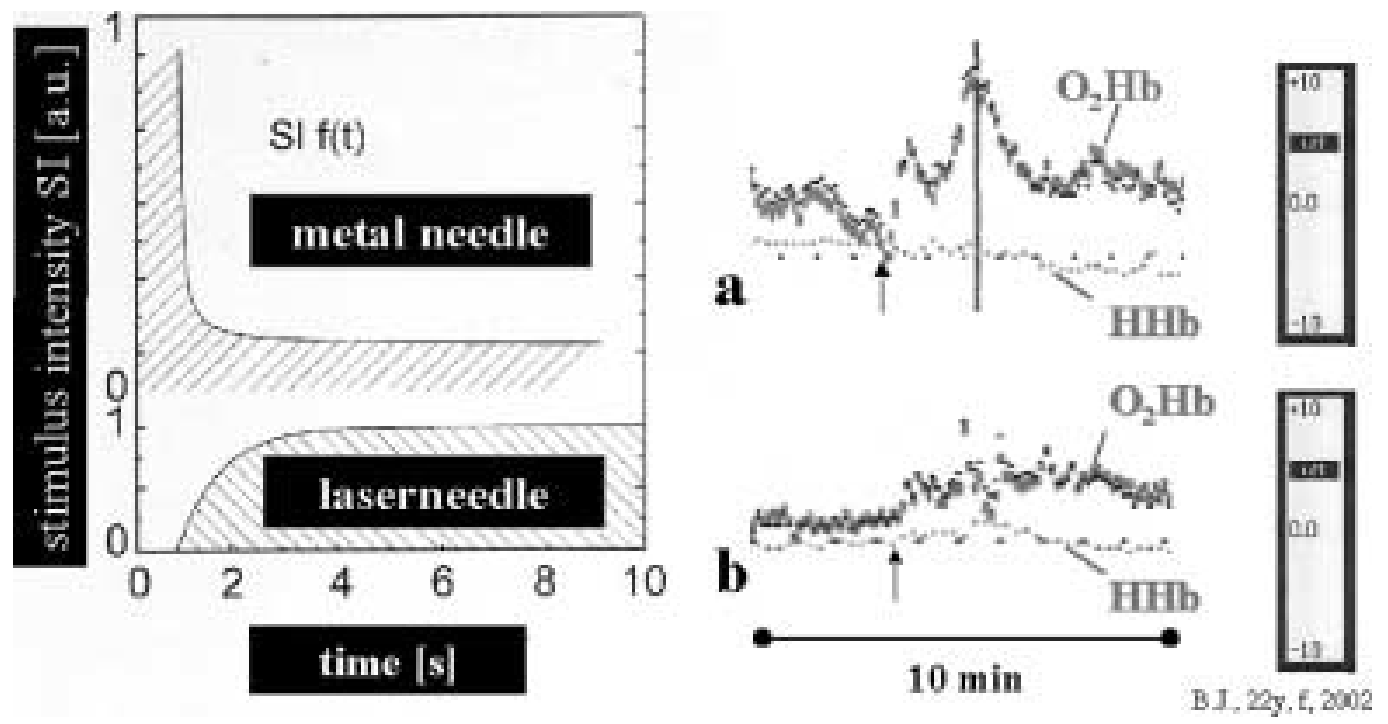

Fig. 3. Left: Stimulus intensity (SI $f(t)$ ) as a function of time (hypothesis). Right: Real measured cerebral responses of NIRS-parameters $\mathrm{O}_{2} \mathrm{Hb}$ (oxyhemoglobin) and $\mathrm{HHb}$ (desoxyhemoglobin) on manual, brief (20 seconds) acupuncture needle stimulation (a) and laserneedle stimulation (b) in 22-year-old female test person. The arrows indicate the beginning of stimulation.

European Marco Polo Comp., Albi, France; hand: $0.1 \times 8 \mathrm{~mm}$, Sooji-Chim, Korea). Stimulation was performed with simultaneous rotating, pulling and thrusting movements of medium intensity.

In the case of laserneedle acupuncture, the skin at the acupuncture point was cleaned with alcohol, the laserneedle was positioned at the surface of the skin and then fixated with special adhesive tape. We used the same acupuncture schemes as in the combined measurements using needle acupuncture.

During the experimental phase, the test persons were positioned in a relaxed manner on a lounge. After applying the near-infrared spectroscopic sensors in the frontal area of the skull, a 10 minute resting period was observed. Then, either laserneedle stimulation was activated or the acupuncture needles were inserted and stimulated for 10 seconds. Thereafter, the laser was activated for 10 minutes or the needles were left alone. The maximum amplitude of $\Delta \mathrm{O}_{2} \mathrm{Hb}$ and $\Delta \mathrm{HHb}$ (phase during acupuncture) was analyzed during this period of time. Randomized selection of which technique should be started with, as well as selection of sequence of the particular type of stimulation (body, ear, hand, combination) was done. The resting period between each investigation was at least 30 minutes.

\subsection{Statistical analysis}

Data was analyzed with the computer program SigmaStat (Jandel Scientific Corp., Erkrath, Germany). Results from the phases before (= zeropoint calibration), during and 5 minutes after needle acupuncture or of laserneedle acupuncture are shown in the diagrams as mean values, respectively.

\section{Results}

At the left side of Fig. 3, the hypothetical functional curve of stimulus intensity dependent upon the treatment time is shown. This diagram gains in importance due to the actually measured, specific cerebral 


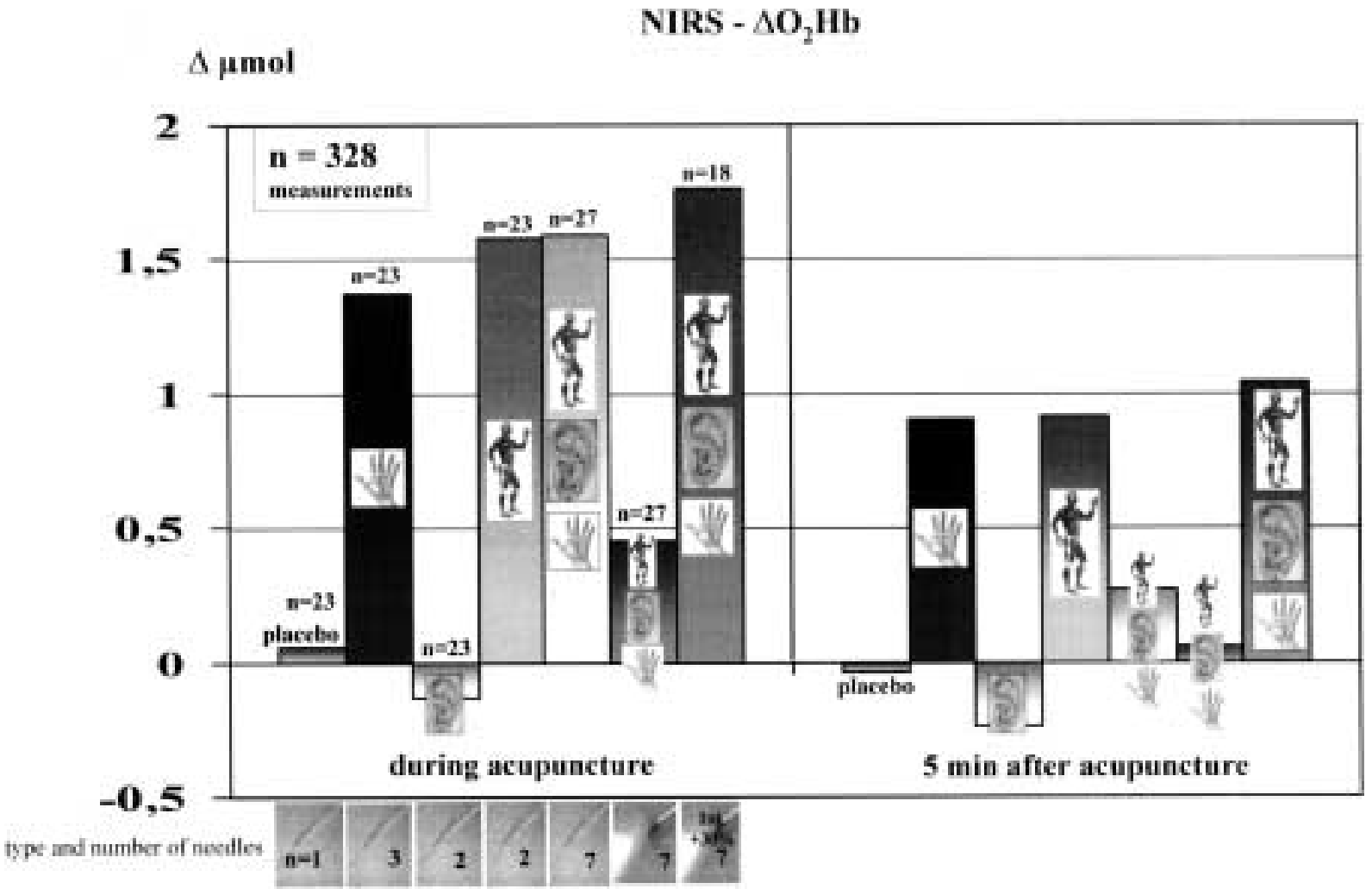

Fig. 4. Changes in $\mu \mathrm{mol}$ of oxyhemoglobin $\left(\mathrm{O}_{2} \mathrm{Hb}\right)$ during needling of a placebo point, 3 hand acupuncture points, 2 ear acupuncture points, 2 acupuncture points from TCM, a combination of hand, ear and body acupuncture as well as laserneedle stimulation and an intensity-increased $(+30 \%)$ laserneedle acupuncture (from left to right) during and 5 minutes after acupuncture.

data in regard to changes in $\mathrm{O}_{2} \mathrm{Hb}$ and $\mathrm{HHb}$ shown at the right. During manual, metal needle stimulation a nearly exponential maximum increase in $\mathrm{O}_{2} \mathrm{Hb}$ and a exponential decrease to a higher level than initially, occurred, whereas the trend of $\mathrm{O}_{2} \mathrm{Hb}$ during laserneedle NIRS response remains plateau-like.

Figures 4 and 5 show the mean values of maximum change in $\mathrm{O}_{2} \mathrm{Hb}$ (Fig. 4) and $\mathrm{HHb}$ (Fig. 5) parameters during and 5 minutes after manual needle acupuncture or laserneedle acupuncture.

It is obvious that needling and stimulation of the placebo point does not lead to marked changes in cerebral NIRS parameters during and 5 minutes after acupuncture. Manual needling and laserneedle stimulation leads to a marked increase in $\mathrm{O}_{2} \mathrm{Hb}$ (compare Fig. 4) and simultaneous decrease in $\mathrm{HHb}$ (compare Fig. 5) when using the combined Korean hand acupuncture (E2) and Chinese hand acupuncture (Yan Dian), as well as TCM-body (Zhanzu and Yuyao) acupuncture, as well as combined body, ear, and hand acupuncture. This effect is still present 5 minutes after removing the needles or deactivating laserneedle stimulation. An almost negligible, but contrary behavior of $\mathrm{O}_{2} \mathrm{Hb}$ and $\mathrm{HHb}$ occurs when both ear points (eye and liver) are needled or stimulated with laser.

None of the acupuncture stimulation methods or combinations resulted in significant changes in standard monitoring parameters (blood pressure). 


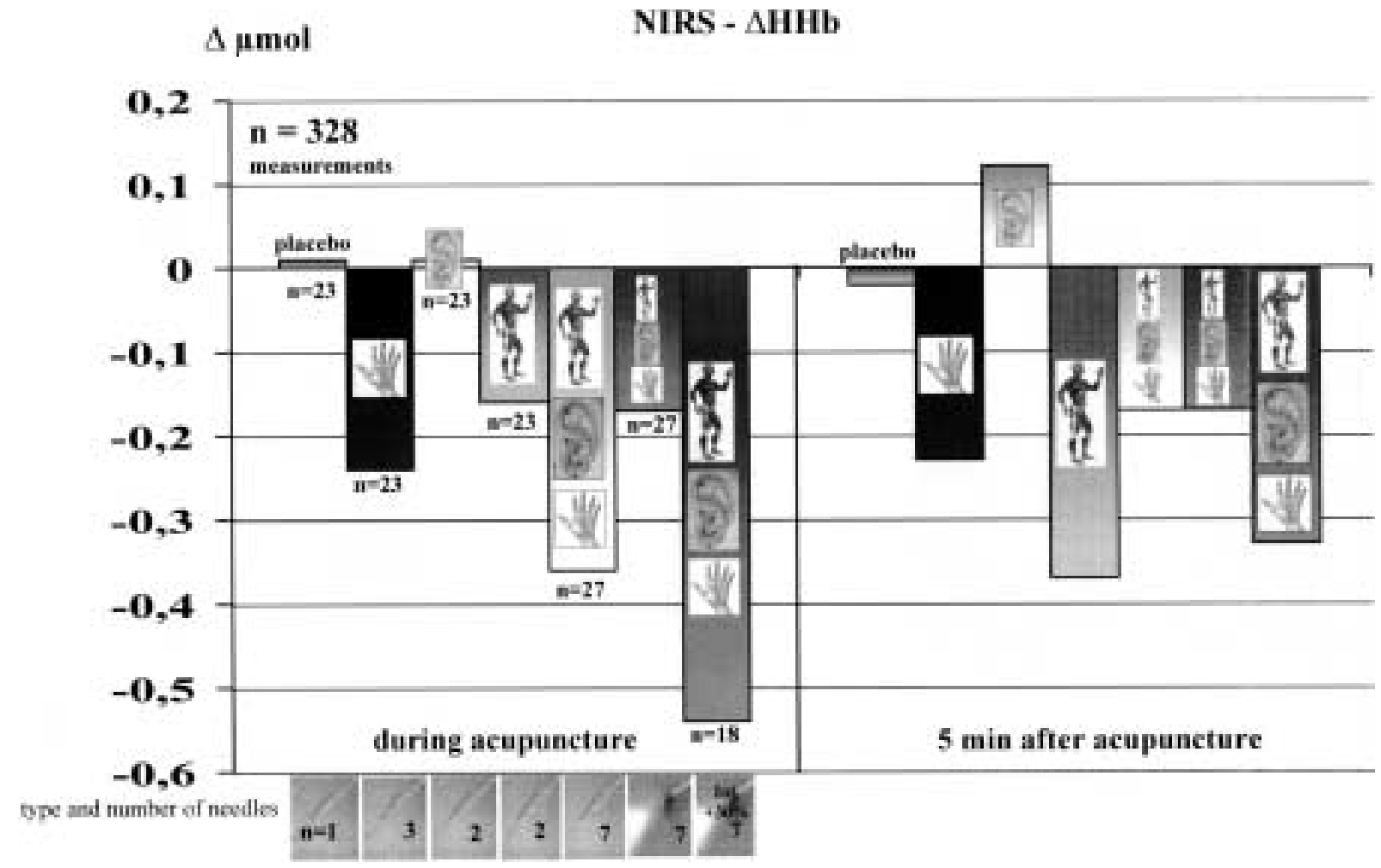

Fig. 5. Cerebral changes (generally decreases) in desoxyhemoglobin (HHb). For further descriptions see Fig. 4.

\section{Discussion}

One of the main advantages of the Laserneedle ${ }^{\circledR}$-technique is its non-invasiveness. It is possible to apply the laser in such a manner, that the test person cannot feel optical stimulation of the acupuncture point. In addition, the acupuncturer does not need to know if the system is activated or deactivated. Thus, double-blind studies using this new method are possible in acupuncture research for the first time. This method of study was already performed by our research group [12] and included simultaneous and continuous monitoring of blood flow velocity in the posterior cerebral artery and the middle cerebral artery in 17 healthy volunteers. This study showed that Laserneedle ${ }^{\circledR}$ stimulation of distant acupuncture points at hands and feet (Hegu, Zusanli, Kunlun, Zhiyin) is able to achieve marked and specific changes in cerebral blood flow velocity $[10,12]$.

Even though laser puncture using low-level-laser stimulation devices is an established method, measurable cerebral effects lie far below conventional needle acupuncture [13]. The results from the first studies $[9,10,12]$ using the Laserneedle ${ }^{\circledR}$ system revealed significant changes in cerebral parameters (blood flow velocities), which were otherwise only achieved by manual needle acupuncture. The proportion of maximum change in blood flow velocity (needle/laserneedle) is approximately factor 2 .

Since Chinese medicine and acupuncture are considered an integrative part of TCM based on energetic processes, the registration of changes in the cerebral metabolism could express energetic processes in the brain and obviously plays a key role in investigating the effects of acupuncture. To date, it has not been possible to obtain non-invasive and continuous results regarding regional cerebral oxygenation. Nearinfrared spectroscopy can register changes in oxygenation in the cerebral vascular region very sensitively. 
The advantages of transcranial oximetry are its non-invasiveness, low risks and continuity, as well as its easy and time-saving application. A wide range of indications are the result for the potential use of this spectroscopic method [4].

A number of factors which can influence adequate interpretation of data must be considered. Contamination with surrounding light, mechanical irritations, intracerebral hematoma, misplacement of optodes or other user errors are just some possibilities which should be noted [4].

A number of studies which deal with NIRS conclude that NIRS can exactly determine extremely small changes in cerebral hemodynamics, as a response to different functional stimulations.

In this study, 328 systematic NIRS registrations on healthy volunteers during manual and laserneedle acupuncture stimulation were performed for the first time. The results from two preceding publications $[6,7]$ were the reference points for this study.

The first study regarding acupuncture and NIRS [6] indicated that the changes in the occipital region after acupuncture stimulation in 3 healthy volunteers, was measurable and reproducible in each of the test persons. In the second study [7], NIRS-changes were measurable and reproducible at the central region after acupuncture stimulation at the Hegu point. This study showed, that reproducible changes in frontally monitored NIRS parameters could be determined, after stimulation of specific eye acupuncture points.

In general, changes in NIRS parameters are unspecific and we do not know if an isolated decrease in saturation is caused by an increase in cerebral oxygenation consumption or results from a decrease in cerebral blood flow. Therefore, not only the extent of oxygenation is shown, but the interaction between oxygenation and desoxygenation is reflected. This is possible since the measurement zone is mainly dominated by the venous part of the cerebral vascular bed $(\sim 75 \%)$. The arterial part $(\sim 20 \%)$ or the capillary $(\sim 5 \%)$ flow region is respectively smaller [4].

For these reasons, we were able to determine changes, which for example, occur due to an increase in oxygenation. Which ruling mechanisms are present is still unclear. Increased desoxygenation by stimulus-induced neuronal activation, i.e., caused by changes in membrane potentials or release of neurotransmitters could be possibilities [14]. For whatever reason, acupuncture obviously influences the oxygen metabolism of the brain in healthy test persons.

Similar to this study using ear acupuncture, a paradox contra-directional change in blood flow velocity (increase) and regional cerebral $\mathrm{O}_{2}$-saturation (decrease) occurred in a vascular based case of dementia, when an individually adapted acupuncture scheme was used [14]. The described case report showed that acupuncture could improve the clinical status of vascular dementia. Using NIRS and transcranial Doppler sonography, we were able to register the effects on cerebral blood flow velocity and the $\mathrm{O}_{2}$-metabolism. In combination with clinical findings, an inverse decrease in regional cerebral $\mathrm{O}_{2}$-saturation during simultaneous increase in cerebral blood flow velocity during acupuncture could be interpreted, as a sign of increased cerebral oxygenation. A decrease in regional cerebral $\mathrm{O}_{2}$-saturation does not necessarily indicate a poor condition of the $\mathrm{O}_{2}$-metabolism in the sense of reduced oxygen supply, however could also document the beneficial effects of regionally increased oxygenation, activated by acupuncture [14]. In a similar manner, the minor contradirectional regional changes in NIRS parameters using ear acupuncture could be interpreted, since the monitoring method conveys the balance between oxygenation and desoxygenation.

Further studies are necessary to investigate the importance of these phenomena on acupuncture, since not only the influence in general and in detail of laser acupuncture, but also the influence of combined ear and body acupuncture, are still discussed controversially. Spectroscopic methods probably are useful tools for this investigations. 


\section{Acknowledgements}

The authors thank Dr. med. Lu Wang for performing the acupuncture, Ms. Evamaria Huber for help in data recording and Mag. Petra Petz for her valuable support in data analysis (all Department of Biomedical Engineering and Research in Anesthesia and Critical Care Graz).

The present report is a pilot study to the presumptive FWF project P16020.

\section{References}

[1] G. Litscher, High-Tech Akupunktur ${ }^{\circledR}$, Pabst Science Publishers, Lengerich, Berlin, Düsseldorf, 2001.

[2] G. Litscher and Z.H. Cho, eds, Computer-Controlled Acupuncture ${ }^{\circledR}$, Pabst Science Publishers, Lengerich, Berlin, Düsseldorf, Riga, Scottsdale, Wien, Zagreb, 2000.

[3] Z.H. Cho, E.K. Wong and J. Fallon, Neuro-Acupuncture, Q-puncture, Los Angeles, 2001.

[4] G. Litscher and G. Schwarz, eds, Transcranial Cerebral Oximetry, Pabst Science Publishers, Lengerich, Berlin, Düsseldorf, Riga, Scottsdale, Wien, Zagreb, 1997.

[5] G. Litscher, G. Schwarz, A. Sandner-Kiesling, I. Hadolt and E. Eger, Effects of acupuncture on the oxygenation of cerebral tissue, Neurol. Res. 20(S1) (1998), 28-32.

[6] G. Litscher and L. Wang, Zerebrale Nahinfrarot-Spektroskopie und Akupunktur - Ergebnisse einer Pilotstudie, Biomed. Technik 45 (2000), 215-218.

[7] G. Litscher, L. Wang and E. Huber, Veränderungen zerebraler nahinfrarot-spektroskopischer Parameter während manueller Akupunkturnadelstimulation, Biomed. Technik. 47 (2002), 76-79.

[8] G. Litscher and L. Wang, Computergestützte Objektivierung der Grenzen der Akupunktur, AKU Akupunktur Theorie und Praxis 30(1) (2002), 13-19.

[9] G. Litscher and D. Schikora, Cerebral effects of noninvasive laserneedles measured by transorbital and transtemporal Doppler sonography, Lasers Med. Sci. 17 (2002), in press.

[10] G. Litscher and D. Schikora, Neue Konzepte in der experimentellen Akupunkturforschung - Computerkontrollierte Laserpunktur (CCL) mit der Laserneedle ${ }^{\circledR}$ Technik, Der Akupunkturarzt/Aurikulotherapeut 3 (2002), 18-28.

[11] Litscher G., Computer-based objectivation of traditional Chinese-, ear- and Korean hand acupuncture, needle-induced changes of regional cerebral blood flow velocity, Neurol. Res. 24 (2002), 377-380.

[12] G. Litscher and D. Schikora, Effects of new noninvasive laserneedles on brain function, EMBEC 2002, 2nd European Medical \& Biological Engineering Conference, Vienna, December 2002, in press.

[13] G. Litscher, L. Wang and M. Wiesner-Zechmeister, Specific effects of laserpuncture on the cerebral circulation, Lasers Med. Sci. 15 (2000), 57-62.

[14] G. Litscher, G. Schwarz, L. Wang and A. Sandner-Kiesling, Akupunktur bei vaskulär bedingtem dementiellen Abbau. Jahrestagung der Österreichischen Alzheimer-Gesellschaft. 14. Klagenfurter Arbeitstagung für Neurologie, 24-25 Mai 2002, Klagenfurt, Austria. 


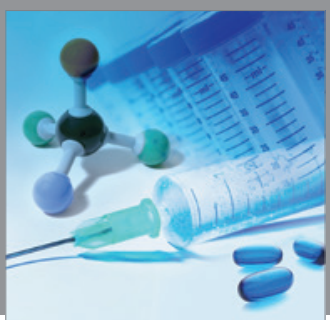

International Journal of

Medicinal Chemistry

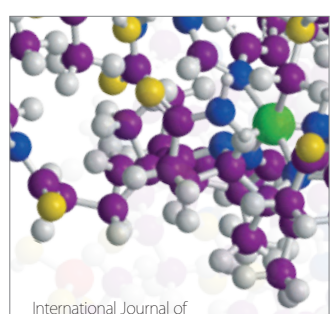

Carbohydrate Chemistry

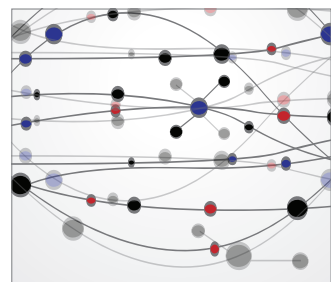

The Scientific World Journal
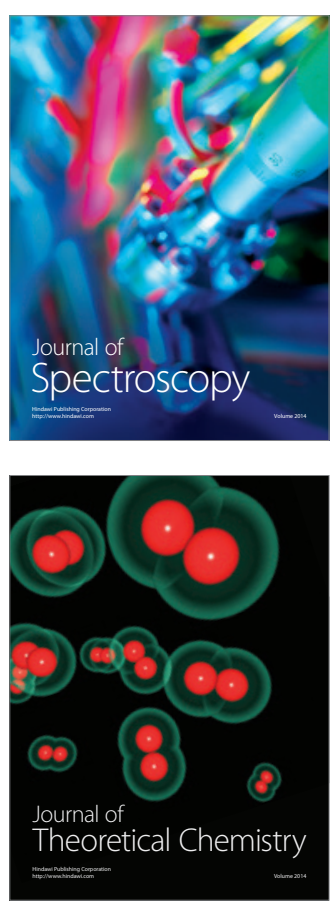
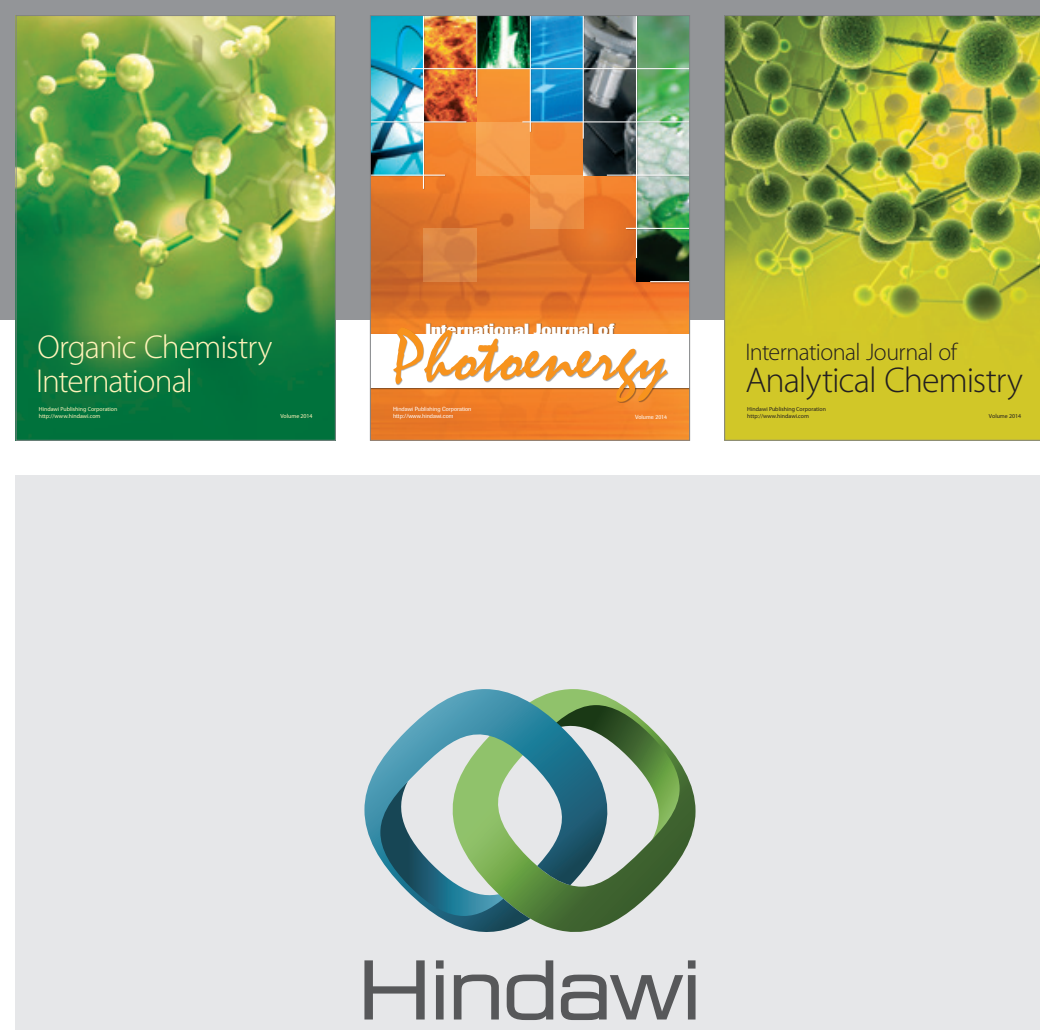

Submit your manuscripts at

http://www.hindawi.com
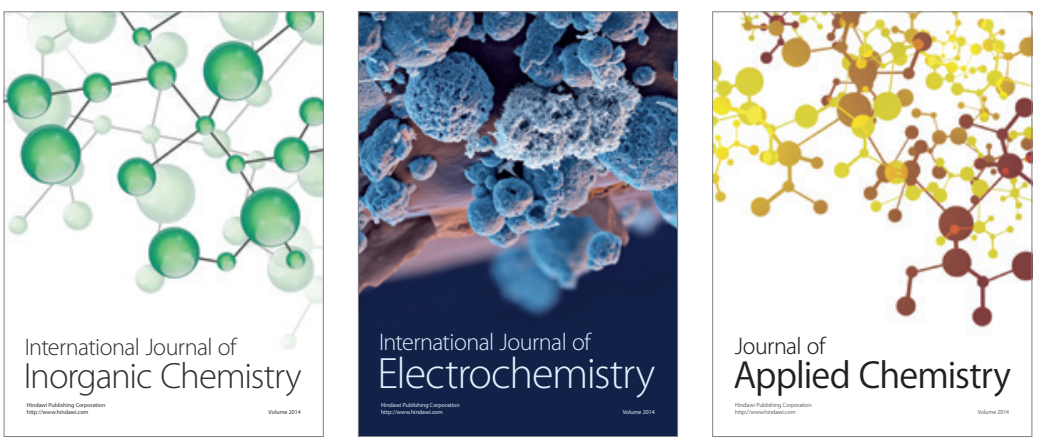

Journal of

Applied Chemistry
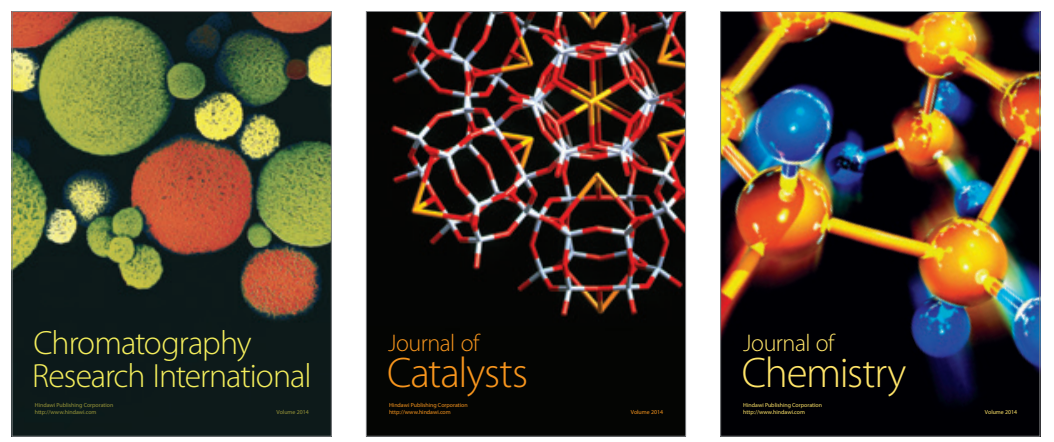
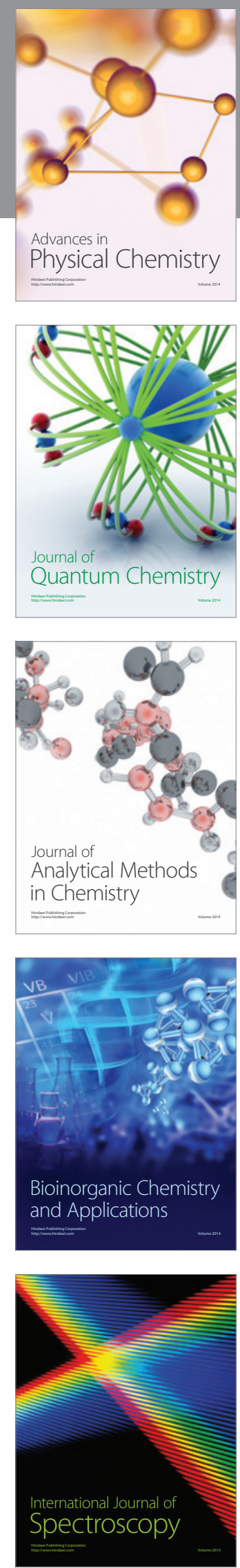